\title{
Vowel productions in the speech of three children with cri du chat syndrome
}

\author{
KRISTIAN EMIL KRISTOFFERSEN \\ Department of Linguistics, University of Oslo, Norway
}

\begin{abstract}
This paper presents findings from a perceptual and acoustic study of vowel productions in the speech of three children with cri du chat syndrome, all raised in Norwegian-speaking communities. It is shown that for all three subjects there is considerable variation in different attempts at producing the same target vowels, but to a varying extent depending on both vowel height and quantity. There is also inter-subject variation. Furthermore, there is considerable acoustic overlap between attempts at producing different target vowels. Finally, only to a limited extent do the vowel productions of the three children form vowel spaces comparable to the vowel space of the target language, but again there is inter-subject variation.
\end{abstract}

Keywords: Cri du chat syndrome, vowel productions, acoustic analysis

\section{Introduction}

Cri du chat syndrome (cdes) is a rare genetic disorder associated with missing material on the short arm of chromosome 5 . Features, which vary considerably from patient to patient, include a high-pitched cry in infancy and childhood, low muscle tone, moderate to profound mental retardation, delayed motor development and delayed linguistic development (Cornish and Pigram, 1996; Cornish and Munir, 1998; Cornish \& al., 1999). Consonant inventories are considerably smaller than the inventories of typically developing children the same age, inventories develop extremely slowly, and consonants are often heavily distorted (Kristoffersen, 2003a, b; 2004).

In a previous study of vowel productions in the speech of one girl with cdes 
(Kristoffersen, 2003c), it was shown that successive attempts at producing the same target vowel varied extensively. However, there was less variation in her attempts at producing the target vowels /i/ and /A/ than other target vowels. Moreover, considerable acoustic overlap was found in her various attempts at producing different target vowels. Finally, a vowel space comparable to the vowel space of the target language gradually emerged over a period of $21 / 2$ years.

The present study expands on this previous study by incorporating data from three additional children with cdcs. The following three questions will be addressed: (1) To what extent is there variation in the subjects' attempts at producing the same target vowel? (2) To what extent is there acoustic overlap in their attempts at producing different target vowels? (3) Do their attempts at producing target vowels form a vowel space comparable to the vowel space of the target language?

\section{Method}

The data are drawn from speech samples elicited by a picture and object naming test, designed partly in cooperation with the parents of the three children. 251 vowels produced by three children have been transcribed in IPA on the basis of perceptual evaluation, carried out by the author. Each vowel has also been described acoustically in terms of F1-F2 plots.

\section{The subjects}

Three children with cdcs, one girl and two boys, participated in the study. All three have been raised in monolingual Norwegian-speaking environments. Subject A is a girl aged 10;8, who speaks in multi-word utterances of some complexity, and who communicates relatively well orally. Subject $\mathrm{B}$ is a boy aged $9 ; 2$ and subject $\mathrm{C}$ is a boy aged 10 years. Both have little spoken language, speaking in one-word utterances intelligible only to those who know them well. One difference between the two boys is that even though subject $\mathrm{C}$ has more spoken language, subject $\mathrm{B}$ uses more consonants with an oral constriction. A typical word in subject C's speech consists of a vowel, occasionally preceded by a glottal stop or fricative, cf. the the following three examples: 


$$
\begin{aligned}
& \text { [uə] for [gur] 'yellow' } \\
& \text { ['?u.Pa] for ['broms.ta] 'flowers' } \\
& \text { ['hœ.œ] for ['nœk.ra] 'keys' }
\end{aligned}
$$

Many of subject B's words, on the other hand, have both onset and coda consonants with an oral constriction, cf.

$$
\begin{aligned}
& \text { ['ygõ.kə] for ['sku:.a] 'shoes-the' } \\
& \text { ['mı.mı] for ['ma.ma] 'mummy' }
\end{aligned}
$$

The target language is minimally different variants of what is called Urban East Norwegian (UEN). UEN has 9 short and 9 long vowel phonemes ${ }^{1}$ (cf. figure 1), which can all appear as nuclei in accented syllables. In unaccented syllables all short vowel phonemes plus schwa can appear.
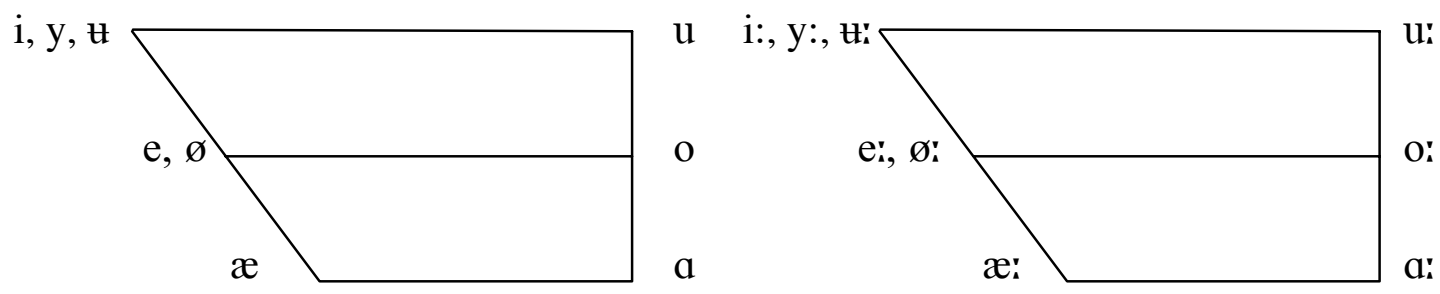

Figure 1. Long and short vowel phonemes in UEN

One aspect of the phonology of Norwegian which deserves mention, is the number of close vowels, in particular the rounded vowels in the front to central area, where there is a contrast between an outrounded /y/ and an inrounded / $\mathfrak{t} /$ (cf. figure 1). At an early stage, typically developing children acquiring Norwegian have problems with the distinction between $/ y /$ and $/ \mathfrak{t} /$, and commonly replaces one with the other (Simonsen 1990). As we will see below, also the subjects participating in the present study have problems with this distinction to varying degrees.

\footnotetext{
${ }^{1}$ In addition UEN has three diphtongs, which are not included in figure 1.
} 


\section{Results}

Short vowels

Table 1 presents the perceptual evaluation of the three subjects' attempts at producing short target vowels.

Table 1. Perceptual evaluation of attempts at producing short target vowels

\begin{tabular}{|c|c|c|c|}
\hline Target vowel & Subject A & Subject B & Subject C \\
\hline i & $\mathrm{i}$ & $\mathrm{i}$, ə & $\mathrm{i}, \partial, \mathfrak{t}, \Theta$ \\
\hline $\mathrm{y}$ & (i) & - & (i) \\
\hline $\mathrm{u}$ & (u) & $(U)$ & $\mathrm{H}, \mathrm{U}$ \\
\hline $\mathrm{u}$ & $\mathrm{u}, \mathrm{o}$ & - & - \\
\hline e & $\mathrm{e}, \varepsilon, \mathrm{I}$ & $\varepsilon, \partial$ & $\varepsilon, \mathfrak{x}, \mathrm{a}$ \\
\hline$\varnothing$ & $\varepsilon, \varnothing, œ$ & - & - \\
\hline $\mathfrak{x}$ & $æ, \varepsilon$ & (æ) & $(æ)$ \\
\hline$a$ & $\mathrm{a}, \mathfrak{x}$ & $\Lambda, \mathfrak{X}$ & $\mathfrak{x}, \partial, \mathrm{a}, \mathrm{U}$ \\
\hline
\end{tabular}

Consider first the vowels produced by subject A. The perceptual evaluations indicate no variation in attempts at target /i/, but variation for the other target vowels. Furthermore, some overlap can be seen between attempts at target vowels /i/ and /e/, and between attempts at target vowels /e/, /ø/, /æ/ and /a/.

The transcriptions based on perceptual evaluation can now be compared with the F1-F2 plots for subject A's short vowels, given in figure 2a.

Insert figure 1 about here

Figure 2a shows, firstly, acoustic variation in the pronunciation of each of the target vowels. Secondly, there is acoustic overlap, in particular among the non-close vowels. And thirdly, to some extent the plots in figure $2 \mathrm{a}$ form a vowel space - defined by the plots for $/ \mathrm{i} /, / \mathrm{e} / \mathrm{and} / \mathrm{u} /$ in the mid to close range, and the plots for $/ \mathfrak{a} / \mathrm{and} / \mathrm{a} /$ in the open range. Also the plots for $/ \varnothing /$ and /o/ occupy different parts of the diagram, but there is considerable overlap between /e/ and / $\varnothing /$ on the one hand, and between /o/ and $/ \mathrm{u} /$ on the other.

Consider next the perceptual evaluations of short vowels in subject B's sample (cf. the third column in table 1). For the three target vowels with more than one token 
each in the material, i.e. /i/, /e/ and /a/, there are two different realizations, one close to the target vowel, and one more centralized variant. There is also overlap between attempts at target vowels /i/ and /e/, and between target vowels /æ/ and /a/. The F1-F2 plots, given in figure $2 \mathrm{~b}$, also clearly show this pattern, with partial overlap between /i/ and /e/, and between the mid and open vowels. The plots in figure $2 \mathrm{~b}$ do not form a vowel space, but note that the plots for /i/ stand out from the other plots.

Finally in this section on the short vowels we consider subject C's productions. The perceptual evaluations (cf. the fourth column in table 1) indicate extensive variation. Target /i/ and /a/, for example, are both realized in four different ways. Also the other short target vowels produced by subject B are realized in several different ways. ${ }^{2}$ In addition, the perceptual evaluations suggest overlap between productions of the target vowels /i, e, æ, a/. These evaluations are confirmed by the F1-F2 plots in figure 2c, showing both extensive variation and extensive overlap. In this chart there is no indication of a vowel space.

\section{Long vowels}

Table 2 presents perceptual evaluations of the three subjects' attempts at producing long target vowels.

Table 2. Perceptual evaluation of attempts at producing long target vowels

\begin{tabular}{|c|c|c|c|}
\hline Target vowel & Subject A & Subject B & Subject C \\
\hline i: & i & $\mathrm{i}$ & $\mathrm{i}$ \\
\hline $\mathrm{y}:$ & $\mathrm{i}, \mathrm{y}$ & (i) & (i) \\
\hline t: & U & - & $\mathbb{H}, U, \Theta$ \\
\hline u: & $\mathrm{u}, \mathrm{o}$ & 0 & $U, \mathbb{H}$ \\
\hline o: & - & 0 & - \\
\hline e: & $\mathrm{e}, \varepsilon, \mathrm{I}$ & - & - \\
\hline$\varnothing:$ & $\varnothing, u$ & $\theta$ & $0, \theta$ \\
\hline æ: & $(æ)$ & ə & $(\mathfrak{x})$ \\
\hline a: & $\mathrm{a}, \mathfrak{x}$ & - & $\mathrm{a}, \partial$ \\
\hline
\end{tabular}

Note: There appears to be no systematic distinction between short and long vowels in the data. Consequently, the symbols representing the subjects' productions are not marked for length.

Consider first the evaluations of the vowels produced by subject A. Firstly, target /y:/

\footnotetext{
${ }^{2}$ The exceptions are of course /y/ and /æ/, with only one realization each.
} 
is realized as both [i] and [y]. Thus, in the speech of this subject we see signs of the distinction between the three front to central close vowels /i, $y, \mathfrak{u} /$ in UEN. As noted above, there are no signs of this distinction in the speech of the two other subjects participating in this study. Secondly, productions of target /u:/, /e:/ and /ø:/ vary considerably, and productions of target /a/ vary between [a] and [æ]. Moreover, there appears to be some overlap between productions of target /i:/ and /e:/ on the one hand, as was the case also with the attempts at short target vowels by this subject. Finally, the transcriptions indicate acoustic overlap between target / $\mathrm{u}: /$ and / $\varnothing: /$.

Insert figure 3 about here

Again, the acoustic analyses confirm the perceptual judgments, se figure 3a. If we compare figure $2 \mathrm{a}$ (short vowels in subject A's speech) with figure $3 \mathrm{~b}$, we see that there is less variation among the long vowels than among the short ones. On the other hand, there is overlap also among these productions, in particular between pronunciations of target /i:/,/y:/ and /e:/, and between / $\mathrm{t}: / / \mathrm{u}: /$ and /ø:/. To some extent these vowels also form a vowel space comparable to that of the target language, defined by the opposition between /i:/-/y:/-/e:/ vs /ut/-/u:/-ø:/ vs /a:/.

Turning next to subject B, the perceptual evaluations (cf. the third column in table 2) again indicate both variation and overlap, in particular between productions of /u:/ and /o:/, both transcribed as [o]. The acoustic analysis, given in figure $3 b$, supports these perceptual judgments, cf. the plots for /u:/ and /o:/, which overlap extensively. Furthermore, it is possible to see at least the beginning of a vowel space, mostly due to the lack of overlap between /i:/ and /y:/ on the one hand and /æ:/ on the other.

Finally, consider subject C's attempts at producing long target vowels. The perceptual evaluations (cf. the fourth column in table 2) indicate extensive variation, with one clear exception - /i:/ and /y:/ are both transcribed as [i]. Furthermore, there appears to be some overlap between rounded close and mid vowels. The acoustic analysis points in the same direction (cf. figure $3 \mathrm{~b}$ ), with extensive variation and overlap. However, also in this case it is possible to trace the beginnings of a vowel space, defined by the plots for /i:/ and / $\mathrm{t}$ // in contrast with the other plots. 


\section{Summary and discussion}

This study of vowel productions in the speech of three children with cri du chat syndrome has shown that there is extensive variation in different attempts at producing the same target vowel. There is also extensive overlap in attempts at producing different target vowels. In the case of overlap, however, there are both inter-subject variation and variation related to the different target vowels. Subject A makes with a few exceptions a clear distinction between the close vowels $/ \mathrm{i}(:) /, / \mathfrak{t}(:) /$ and $/ \mathrm{u}(:) /$ (figures $2 \mathrm{a}$ and $3 \mathrm{a}$ ), whereas subject $\mathrm{B}$ makes a distinction between $/ \mathrm{i} / \mathrm{and}$ /a/ in his attempts at the short target vowels (figure $2 \mathrm{~b}$ ) and between /i:/ and /u:/ in his attempts at the long target vowels (figure $3 b$ ). Subject $\mathrm{C}$ makes a distinction between /i:/ and the rest of the long vowels (figure 3c). No such distinction is apparent among this subject's attempts at producing short target vowels (figure 2c). In other words, all three subjects make relatively clear distinctions in their attempts at producing long target vowels, only two of them (A and B) do the same for short vowels.

Note that the vowel that for all subjects stands out from all other vowels is /i(:)/. This was also the case with the girl whose speech was reported in Kristoffersen (2003c). Furthermore, the distinctions that are being made, that is, /i/ vs / $\mathfrak{u} /-/ \mathrm{u} / \mathrm{vs}$ /a/ conform relatively well with the simple phonological systems which are seen in early child language.

These findings raise a couple of important questions, the first of which is concerned with the distinction between delayed and deviant language. Obviously, all three subjects have extremely delayed vowel productions compared to typically developing Norwegian-speaking children. Unfortunately, no standardized data providing a basis for comparison exist for Norwegian, but the database in Simonsen (1990, appendix II) clearly suggests that typically developing children between two and three years acquiring Norwegian have far more developed vowel systems than any of the three subjects who participated in the present study.

What is more striking with the vowel productions discussed here, however, is their deviant character. Remember that the three subjects who participated in this study are between nine and ten years, and even though vowel productions of typically developing children are more variable than adult vowel productions (Lee, Potamianos, 
and Narayanan, 1999), the findings from the present study suggest variation to such a degree that it constitute a barrier to effective communication. Thus, the study supports numerous observations that patients with cri du chat syndrome experience severe problems with communicating orally. In particular, it has been shown that one source of these problems are overlapping vowel productions, which together with the reduced consonant inventories associated with cri du chat syndrome (Kristoffersen, 2003a, 2004) inevitably result in extensive homonymy. One example of this homonymy is subject C's form ['?U.?a], which express two different meanings in this sample, namely 'flowers' (for target [['broms.ta]) and 'apple' (target = [æp.rə].

This raises the further question whether the observed deviations are phonetic or phonological in nature. This is of course difficult to answer without further empirical investigations. However, patients with cri du chat syndrome have motor problems to various degrees, resulting among other things in difficulties in controlling muscles involved speech production (cf. Kristoffersen, 2003a, b, 2004). The extreme acoustic overlap seen in the vowel productions discussed above may well be the consequence of these motor problems, more specifically problems with controlling the muscles involved in production of vowels. It is also possible that some of these overlapping productions have been phonologized. Resolving this question, however, will have to be left for future research.

\section{References}

Cornish, K., Bramble, D., Munir, F. and Pigram, J., 1999, Cognitive functioning in children with typical cri du chat (5p-) syndrome. Developmental medicine and child neurology, 42, 263-266.

Cornish, K. and MuniR, F., 1998, Receptive and expressive language skills in children with cri du chat syndrome. Journal of communication disorders, $\mathbf{3 1}$, 73-81.

CORNISH, K. and PIGRAM, J., 1996, Developmental and behavioural characteristics of cri du chat syndrome. Archives of disease in childhood, 75, 448-450.

KRISTOFFERSEN, G., 2000, The phonology of Norwegian (Oxford: Oxford University Press). 
KRISTOFFERSEN, K. E., 2003a, Development of consonants and vowels in a child with cri du chat syndrome. Journal of Multilingual Communication Disorders, 1, 194-200.

Kristoffersen, K. E., 2003b, Phonological development in a child with cri du chat syndrome. Nordlyd, 31.3, 519-531. (http://www.ub.uit.no/munin/nordlyd/)

KRISTOFFERSEN, K. E., 2003c, Vowel production in cri du chat-syndrome - results from a case study. In M. J. Solé, D. Recasens and J. Romero (eds.), Proceedings from The 15th International Congress of Phonetic Sciences (Adelaide, Australia: Causal Productions Pty Ltd), pp. 1663-1666.

KRISTOFFERSEN, K. E. 2004, Consonant production in three children with Cri du chat syndrome. In B.E. Murdoch, J. Goozee, B.-M. Wehlan \& K. Docking (Eds.): 2004 IALP Congress - Proceedings.

Lee, S, Potamianos, A, and Narayanan, S. 1999, Acoustics of childrens's speech: Developmental changes of temporal and spectral parameters. Journal of the Acoustical Society of America, 105, 1455-1468. 


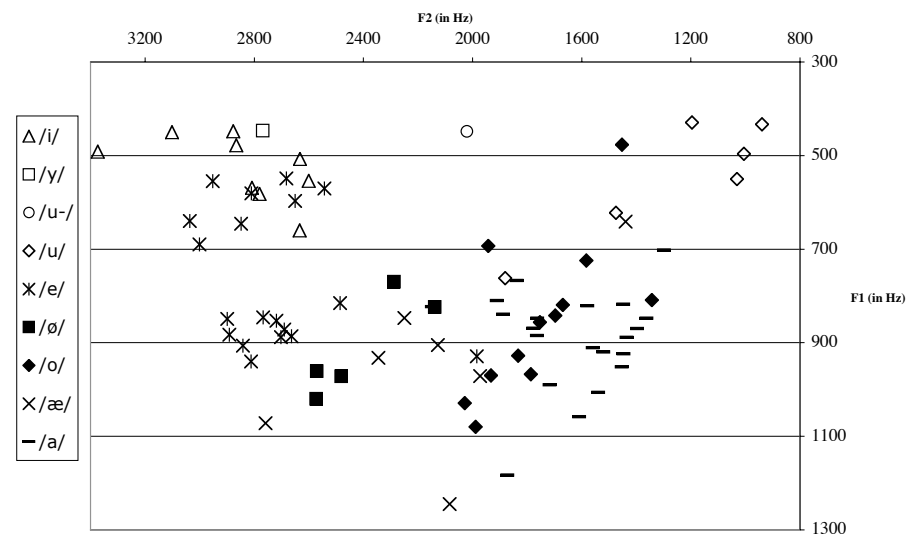

(2a) F1-F2 plots of subject A's short vowels

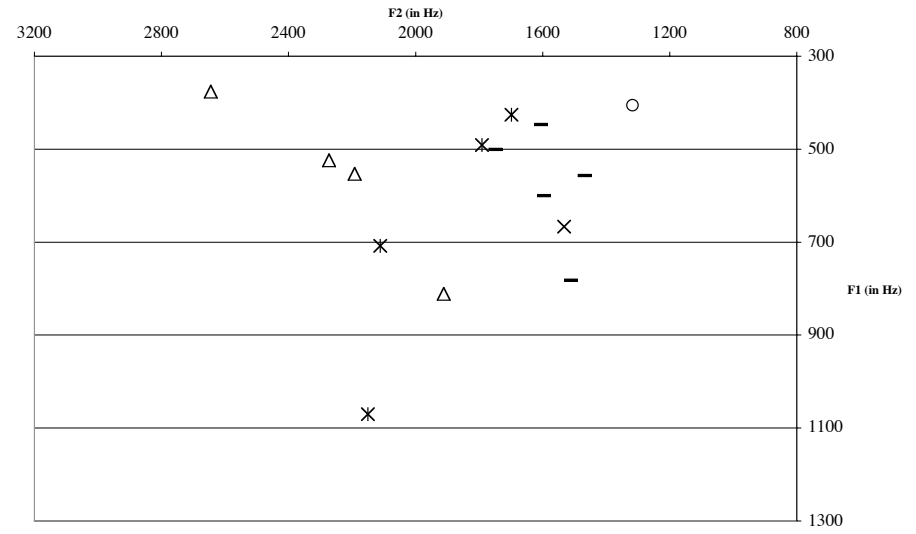

(2b) F1-F2 plots of subject B's short vowels

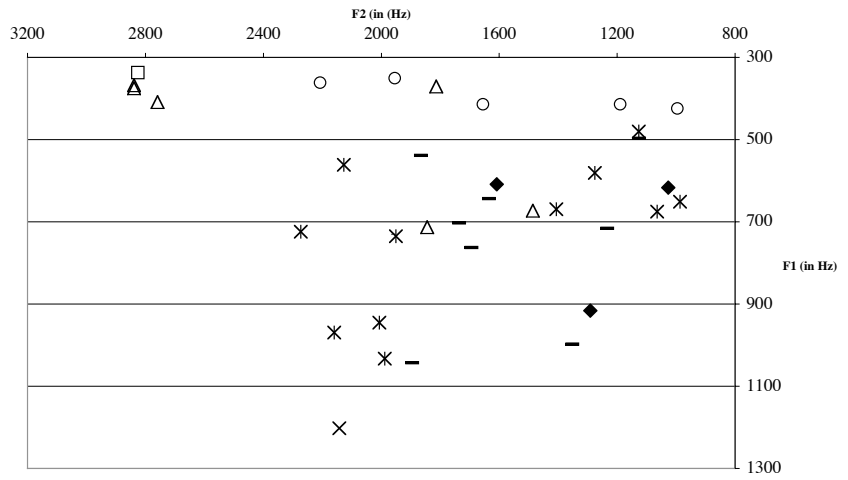

(2c) F1-F2 plots of subject C's short vowels

Figure 2. F1-F2 plots of short vowels 


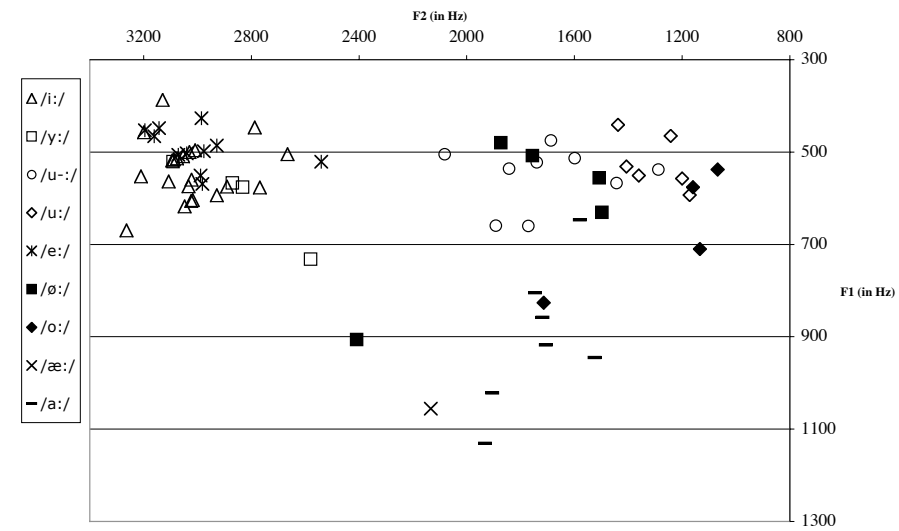

(3a) F1-F2 plots of subject A's long vowels

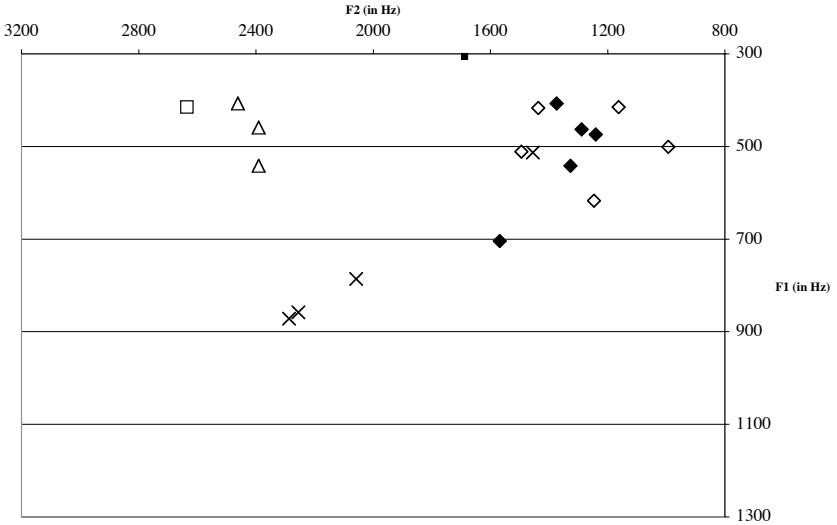

(3b) F1-F2 plots of subject B's long vowels

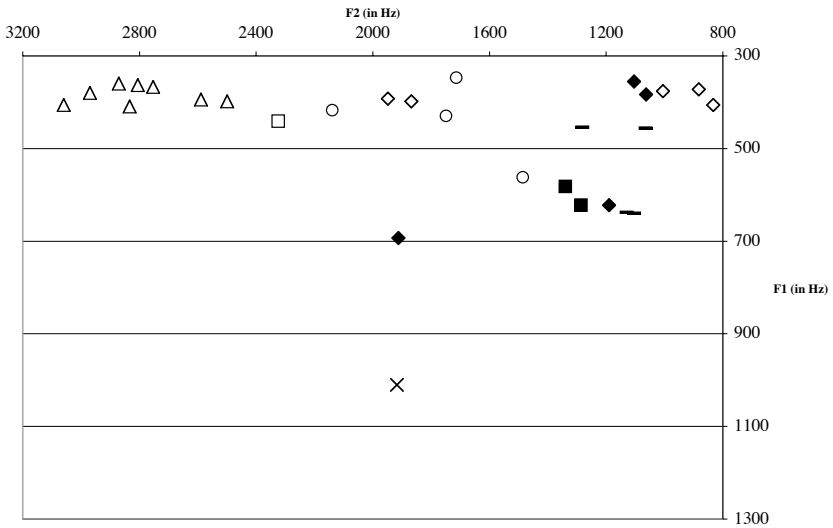

(3c) F1-F2 plots of subject C's long vowels

Figure 3. F1-F2 plots of long vowels 